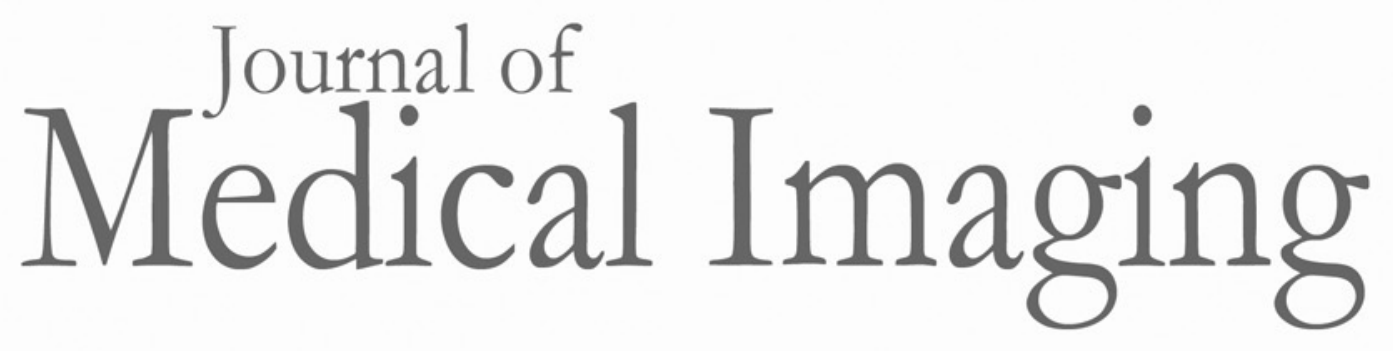

\title{
Evaluation of an iterative model- based reconstruction algorithm for low-tube-voltage (80 kVp) computed tomography angiography
}

Peter B. Noël

Thomas Köhler

Alexander A. Fingerle

Kevin M. Brown

Stanislav Zabic

Daniela Münzel

Bernhard Haller

Thomas Baum

Martin Henninger

Reinhard Meier

Ernst J. Rummeny

Martin Dobritz 


\title{
Evaluation of an iterative model-based reconstruction algorithm for low-tube-voltage $(80 \mathrm{kVp})$ computed tomography angiography
}

\author{
Peter B. Noël, ${ }^{a, \star}$ Thomas Köhler, ${ }^{b}$ Alexander A. Fingerle, ${ }^{a}$ Kevin M. Brown, ${ }^{c}$ Stanislav Zabic, ${ }^{c}$ Daniela Münzel, ${ }^{a}$ \\ Bernhard Haller, ${ }^{d}$ Thomas Baum, ${ }^{a}$ Martin Henninger, ${ }^{a}$ Reinhard Meier, ${ }^{a}$ Ernst J. Rummeny, ${ }^{a}$ and Martin Dobritz ${ }^{a}$

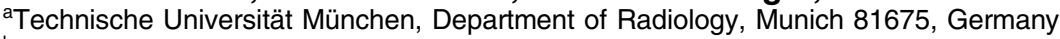 \\ ${ }^{b}$ Philips Research Laboratories, Hamburg 22335, Germany \\ 'Philips Healthcare, Cleveland, Ohio 44143, United States \\ ${ }^{\mathrm{d} T e c h n i s c h e}$ Universität München, Institute of Medical Statistics and Epidemiology, Munich 81675, Germany
}

\begin{abstract}
The objective of this study was to investigate the improvement in diagnostic quality of an iterative model-based reconstruction (IMBR) algorithm for low-tube-voltage (80-kVp) and low-tube-current in abdominal computed tomography angiography (CTA). A total of 11 patients were imaged on a 256-slice multidetector computed tomography for visualization of the aorta. For all patients, three different reconstructions from the low-tube-voltage data are generated: filtered backprojection (FBP), IMBR, and a mixture of both IMBR + FBP. To determine the diagnostic value of IMBR-based reconstructions, the image quality was assessed. With IMBRbased reconstructions, image noise could be significantly reduced, which was confirmed by a highly improved contrast-to-noise ratio. In the image quality assessment, radiologists were able to reliably detect more third-order and higher aortic branches in the IMBR reconstructions compared to FBP reconstructions. The effective dose level was, on average, $3.0 \mathrm{mSv}$ for $80-\mathrm{kVp}$ acquisitions. Low-tube-voltage CTAs significantly improve vascular contrast as presented by others; however, this effect in combination with IMBR enabled yet another substantial improvement of diagnostic quality. For IMBR, a significant improvement of image quality and a decreased radiation dose at low-tube-voltage can be reported. $\odot$ The Authors. Published by SPIE under a Creative Commons Attribution 3.0 Unported License. Distribution or reproduction of this work in whole or in part requires full attribution of the original publication, including its DOI. [DOI: 10.1117/1 .JMI.1.3.033501]
\end{abstract}

Keywords: computed tomography; iterative reconstruction; low-tube-current; computed tomography angiography; dose reduction; low-tube-voltage.

Paper 14045RR received Apr. 15, 2014; revised manuscript received Sep. 3, 2014; accepted for publication Sep. 11, 2014; published online Oct. 9, 2014.

\section{Introduction}

Computed tomographic angiography (CTA) of the abdomen is a well-established procedure for visualization of the abdominal vasculature, specifically the aorta, renal arteries, and splanchnic circulation. $^{1-4}$ One important aspect of abdominal CTA is the vasculature-to-background contrast. This iodine-based contrast can be increased when applying low-tube-voltages. This increased absorption of iodine is caused by a high-photoelectric effect compared to Compton scattering. ${ }^{5}$ The highest benefit in iodine contrast can be achieved when reducing the mean energy closer to the $K$-edge of iodine ( $33 \mathrm{keV})$. Note that the $K$-edge of a material describes a sudden increase in attenuation. This effect occurs when the energy of the incoming x-rays exceeds that of the $K$-shell of the atoms, so that the electrons can be ejected from the atom. On the contrary, when reducing to a low-tubevoltage, the improved iodine signal appears at the cost of a significantly increased image noise. In previous work, this image quality issue was resolved by increasing the tube current, in some reports up to $700 \mathrm{~mA} .^{5-9}$ The low-tube-voltage/hightube-current technique has proven to improve the diagnostic quality of CTAs; however, no significant dose reduction has been achieved. At the same time, the ideal procedure would be to image with low-tube-voltage/low-tube-current in order

*Address all correspondence to: Peter B. Noël, E-mail: peter.noel@tum.de to significantly reduce the radiation exposure while increasing the iodine contrast.

The most promising and currently available technology to reduce the radiation dose in computed tomography (CT) is the use of iterative reconstruction methods ${ }^{5,10-15}$ in combination with the reduction of tube voltage or tube current. Unfortunately, the details of iterative reconstruction methods, which are available on commercial CT systems, are not open to the public. However, the main components are known: first of all, these methods claim to consider the individual noise in each detector channel properly. More advanced methods also claim to obtain further improvements by modeling the system geometry and the system physics more accurately in a feedback-loop scheme than is possible with filtered back-projection type algorithms. We denote, in this work, the second type of algorithms: iterative model-based reconstruction (IMBR).

The principal aim of this study was to evaluate the effect of low-tube-voltage $(80 \mathrm{kVp})$ and low-tube-current in combination with iterative model-based reconstruction.

\section{Methods and Materials}

\subsection{Patient Population}

This single-center study was institutional review board approved. For each participant, a written consent was obtained, as approved by the ethical committee at the Faculty of Medicine 
of the Technische Universität München. The study included 11 patients in an abdominal aortic aneurysm follow-up (three patients with an aortic stent), who underwent an abdominal CTA examination. Patients' younger than 60 years, pregnant, or with history of anaphylactic reaction or renal-failure were excluded from the study.

\subsection{Computed Tomography Acquisition}

All patients were imaged on a 256-slice CT scanner (Brilliance iCT, Philips Healthcare, Cleveland, Ohio) for visualization of the aorta. The departmental CTA protocol was employed; this procedure involves a body mass index (BMI) adjustment of the tube current. The tube voltage of $80 \mathrm{kVp}$ was deviated from the standard clinical protocol $(120 \mathrm{kVp})$, whereas the tube current was maintained in the regular range (80 to $250 \mathrm{mAs}$ ). Patients were placed in the supine position with their feet first on the scanner couch. After an anteroposterior and mediolateral scout to define the optimal scan region, $60 \mathrm{ml}$ of intravenous contrast agent (Iomeron $400 \mathrm{MCT}$, Bracco Imaging Deutschland $\mathrm{GmbH}$, Konstanz, Germany) was injected with an injection rate of $3.5 \mathrm{ml} / \mathrm{s}$ by using a dual syringe injection system (Stellant, MEDRAD, Inc., Indianola, Pennsylvania). The bolus tracker with a regions-of-interest (ROI) placed in the upper abdominal aorta was used to ensure optimal contrast enhancement and to start scanning craniocaudally with a pitch of 0.9 and a $128 \times 0.625-\mathrm{mm}$ detector configuration.

\subsection{Filtered Backprojection}

The standard filtered backprojection (FBP) algorithm of the iCT was used for reference reconstruction using 1-mm slice thicknesses and a medium soft filter. Note that this a standard filter which is routinely used for clinical CTA acquisitions in our department.

\subsection{Iterative Model-Based Reconstruction}

The IMBR algorithm used in this study was a prototype research implementation of a penalized maximum log-likelihood algorithm. ${ }^{16-18}$ Specifically, during reconstruction, the following cost function is minimized

$\Delta(u)=-L(A u \mid y)+\beta R(u)$,

where $u$ is the image, $A$ the system matrix, $y$ is the measured line integrals, and $R$ is a roughness penalty. A Gaussian model is used for the log-likelihood function $L$, i.e.,

$L(A u \mid y)=-\sum_{i} w_{i}^{2}\left(y_{i}-\sum_{j} a_{i j} u_{j}\right)^{2}$,

where the elements of the system matrix $A$ are denoted as $a_{i j}$, the image elements as $u_{j}$, and the individual measured line integrals $y_{i}$. The statistical weights $w_{i}$ are derived from the statistics of the incoming x-ray flux. The roughness $R$ penalty has the form

$R(u)=\sum_{j} \sum_{k \in N_{j}} v_{k} \psi\left(u_{j}-u_{k}\right)$

where the outer sum runs over all image elements $k$ and the inner sum over all 26 neighboring image elements. For the potential function, $\psi_{\delta}$ a Huber potential was used given by $\psi_{\delta}(x)= \begin{cases}x^{2} & |x|<\delta \\ 2 \delta|x|-\delta^{2} & |x| \geq \delta\end{cases}$

which provides a so-called edge-preserving regularization on the images. For all reconstructions, a fixed $\delta$ of $2 \mathrm{HU}$ was used and the distance weights $v_{k}$ are the inverse Euclidian distances between the image points. Optimization of the regularized log-likelihood function was performed using an ordersubset separable paraboloidal surrogate algorithm, see Ref. 18 .

The user defined the trade-off point between the data fidelity term and the roughness term. This was done by a specification of a desired target noise level of the reconstructed image; ${ }^{19}$ the images shown here correspond to $10 \%$ of the FBP noise. Further, to offer a noise texture closer to the standard FBP reconstruction, we generated an extra dataset by fading a certain fraction of the FBP image to the IMBR result (IMBR + FBP); here 5\% FBP fading was used. Please note that IMBR is not commercially available and is a prototype for research proposes only. IMBR is a nonlinear reconstruction algorithm. Therefore, the spatial resolution is-as opposed to linear FBP-type algorithms - object dependent. In particular, due to the use of an edge-preserving regularization, the sharpness of edges depends on their contrast. Consequently, a task-based image quality evaluation is the method of choice for comparison.

\subsection{Image Evaluation}

Subjective image assessment was performed by four radiologists with an average of 2 years of experience (ranging from 1 to 4 years). For each protocol and patient, the full batch of images (on average 1001 slices) is presented in axial and coronal orientation. In total 66 (11 patients, 3 reconstruction algorithms, and 2 view planes) full batches of images are evaluated in random order. Each radiologist individually performed the assessment by ranking one after the other dataset on a diagnostic workstation. Due to the increased iodine contrast, a CTA window setting of center: $200 \mathrm{HU}$ and width: $1200 \mathrm{HU}$ was chosen for diagnostic evaluation. The radiologists did have experience with commercially available iterative reconstruction algorithms, but they did not have previous experience with IMBR reconstructed data. Before starting the rating of the images, the different quality metrics were discussed with each radiologist. This step was done to insure a common understanding for the different metrics. All four radiologists independently rated the following image quality metrics. Images were addressed for subjective image noise on a 4-point scale $(1=$ minimal image noise, $2=$ less than average noise, $3=$ above average noise, $4=$ unacceptable image noise); image artifacts on a 4-point scale $(1=$ no artifacts, $2=$ minor artifacts not interfering with diagnostic decision making, 3 = major artifacts affecting visualization of major structures but diagnosis still possible, $4=$ artifacts affecting diagnostic information); diagnostic confidence on a 4-point scale $(1=$ completely confident, $2=$ probably confident, $3=$ confident only for a limited clinical entity, $4=$ poor confidence); image impression on a 4-point scale $(1=$ natural, $2=$ limited natural, $3=$ limited artificial, $4=$ artificial); and with respect to vascular detectability of third-order and higher aortic branches on a 4 -point scale $(1=$ detectable, $2=$ probably detectable, $3=$ limited detectability, 4 = poor detectability). Kappa values were calculated to compare interobserver agreement on a per-patient basis for all image quality metrics.

In order to remove interpatient variability in our results, we introduce a histogram of differences. For this representation of 
our results, we set the FBP rating as the reference and evaluated the difference of IMBR and IMBR + FBP to the reference score. Since the reference FBP rating was taken from the same reader, this procedure also removes to some extent the inter-reader variability, and thus gives a clear view on the question whether IMBR or IMBR + FBP is superior to FBP. Differences were calculated over all observers and protocols and then combined into a barplot with regard to specific image metrics. The inspection of such a barplot with regard to symmetry, the deviation of its center from zero and the presence of a tail on either side of its center gives a fast simple overview of the results.

For objective image assessment, we measured the image noise and the contrast-to-noise ratio (CNR). For data collection, all images were displayed in axial representation right next to each other. We obtained the mean and standard deviation of $\mathrm{CT}$ attenuation values (in Hounsfield units) at different ROIs. All ROIs were manually drawn at the following regions: (1) aorta, (2) paraspinal muscle, and (3) subcutaneous fat. For the aorta, the ROIs were drawn filling the full lumen while avoiding plaques or stents. All ROIs were placed in regions without helical or windmill artifacts, streak artifacts, or beam hardening artifacts. The CNR was calculated for aorta versus paraspinal muscle and aorta versus subcutaneous fat by:

$\mathrm{CNR}=\frac{\left|\mu_{s}-\mu_{b}\right|}{\sigma_{b}}$,

where $\mu_{s}$ is the mean of the aorta ROI, and $\mu_{b}$ and $\sigma_{b}$ are the mean and the standard variation of the paraspinal muscle or of the subcutaneous fat ROI. Note that due to the use of a nonlinear image reconstruction method noise, contrast and spatial resolutions are dependent on the imaged object and its surrounding tissue. A CNR measured at a particular location does not give information about the situation elsewhere. Thus, this should be considered when reviewing the results in this manuscript.

For dose assessment, the dose length product (DLP) of all patients was recorded. The DLP is a measure of total radiation exposure for the whole series of images. For estimation of the effective dose, the DLP values were converted with a conversion factor of $0.014 \mathrm{mSv} /(\mathrm{mGy} * \mathrm{~cm}){ }^{20}$

\section{Results}

Figure 1 shows the coronal tomographic images of a 72-year-old male patient with an aneurysm of about $6 \mathrm{~cm}$ after placement of an abdominal stent. Panel A shows a preliminary examination with high-tube-voltage $(120 \mathrm{kVp})$ and an effective dose of $8.82 \mathrm{mSv}$, which took place 14 months before the study. Panel B presents the mixture of both IMBR + FBP reconstructions, panel $\mathrm{C}$ is the IMBR reconstruction, and panel $\mathrm{D}$ is the FBP reconstruction. The effective dose of the low-tube-voltage (80 kVp) examination was $1.86 \mathrm{mSv}$ (panels $\mathrm{B}, \mathrm{C}$, and D). We observed that the anatomical and pathological morphologies are more visible with IMBR-based reconstruction compared to the conventional FBP reconstruction. Subjectively, the diagnostic quality may rise above the preliminary examination because of the increased iodine contrast and the image noise is reduced substantially. Figure 2 illustrates the same patient as in Fig. 1 in an axial orientation. Panel A shows the preliminary examination, panel $\mathrm{B}$ is the mixture of both IMBR + FBP reconstruction, panel $\mathrm{C}$ is the IMBR reconstruction, and panel $\mathrm{D}$ is the FBP reconstruction. The white marked and enlarged area focuses on the aneurysm and includes an endoleak. Clearly, the endoleak is visible in panels $\mathrm{A}$ to $\mathrm{C}$, but is harder to detect in the FBP reconstruction of the low-tube-voltage data. Figure 3 shows axial-tomographic images of a 62-year-old male patient with an aortic dissection. Panel A illustrates the mixture of both IMBR + FBP reconstructions, panel B is the IMBR reconstruction, and panel $\mathrm{C}$ is the FBP reconstruction. Note that for this patient, no preliminary examination was available. The effective dose for this acquisition was $2.32 \mathrm{mSv}$. The strongly eliminated noise enables the detection of the aortic dissection in panels $\mathrm{A}$ and $\mathrm{B}$, while it is hard to detect in panel $\mathrm{C}$.

These visual results are confirmed by the subjective image assessment. Table 1 summarizes the results over all cases and observers. Figure 4 illustrates the histogram of differences (as defined in the method section) for "IMBR minus FBP"- - axial (Panel A), "IMBR minus FBP"- coronal (Panel B), "IMBR+ FBP minus FBP"-axial (Panel C), and "IMBR + FBP minus

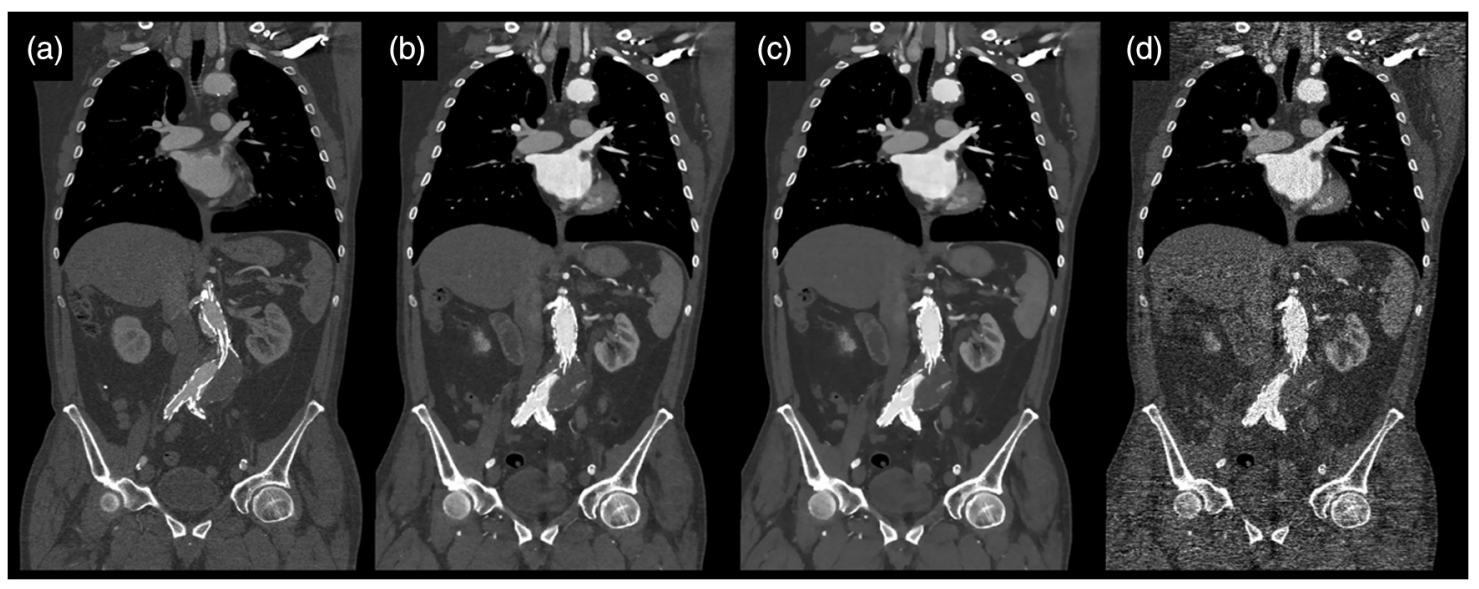

Fig. 1 A 72-year-old male patient with an aneurysm of about $6 \mathrm{~cm}$ after placement of an abdominal stent. The panels (A-D) from left to right: preliminary FBP, IMBR + FBP, IMBR, and FBP reconstruction. The effective dose of the low-tube-voltage $(80 \mathrm{kVp})$ examination was $1.86 \mathrm{mSv}$ (panels $\mathrm{B}, \mathrm{C}$, and $\mathrm{D}$ ), compared to $8.82 \mathrm{mSv}$ with high-tube-voltage (120 kVp) (panel A). (window width, $1200 \mathrm{HU}$; window center, $200 \mathrm{HU}$ ) 


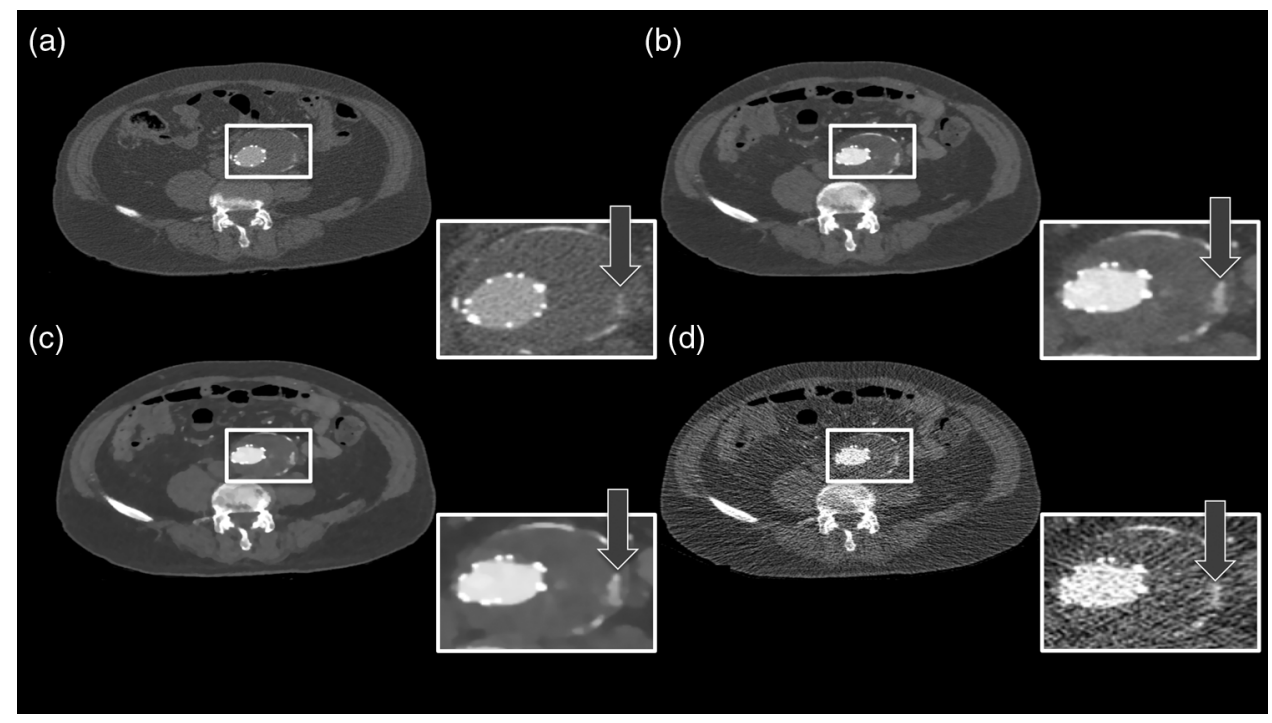

Fig. 2 Same patient as in Fig. 1. Panel A shows the preliminary examination, panel $B$ the mixture of both IMBR + FBP reconstruction, panel C the IMBR reconstruction, and panel D the FBP reconstruction. The white marked and enlarged area focuses on the aneurysm and includes an endoleak. The endoleak is visible in panels $\mathrm{A}$ to $\mathrm{C}$, but significantly harder to detect in the filtered backprojection (FBP) reconstruction. (window width, $1200 \mathrm{HU}$; window center, $200 \mathrm{HU}$ )

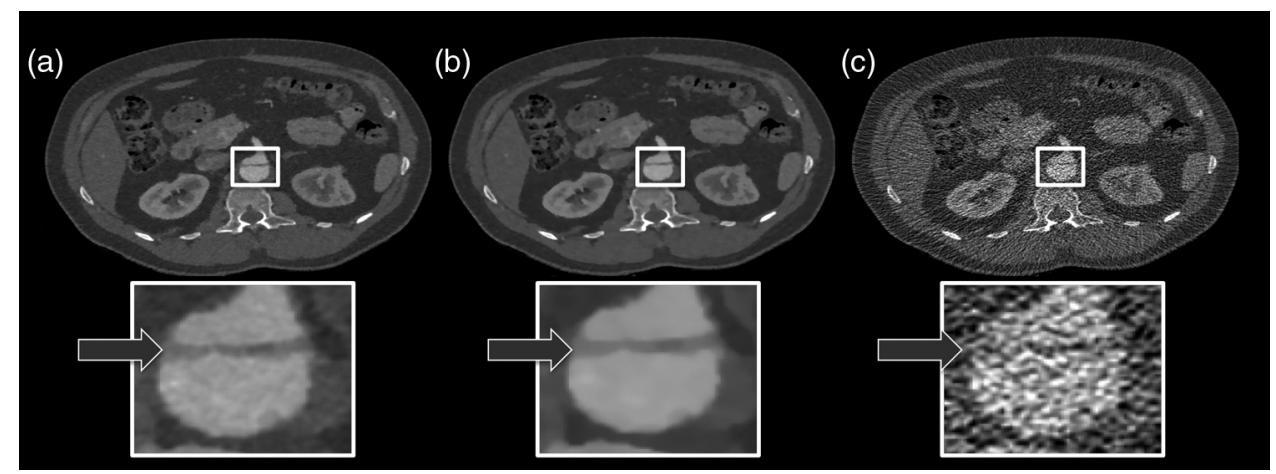

Fig. 3 62-year-old male patient with an aortic dissection. The panels $(A-C)$ from left to right: IMBR + FBP, IMBR, and FBP reconstruction. The effective dose was $2.32 \mathrm{mSv}$. The strongly eliminated noise enables the detection of the aortic dissection in panels $A$ and $B$, while being hard to detect in panel C. (window width, $1200 \mathrm{HU}$; window center, $200 \mathrm{HU}$ )

Table 1 Summary of the subjective image assessment.

\begin{tabular}{|c|c|c|c|c|c|c|c|c|c|c|c|}
\hline \multirow[b]{2}{*}{ Presentation plane } & \multirow[b]{2}{*}{ Recon algorithm } & \multicolumn{2}{|c|}{ Image noise } & \multicolumn{2}{|c|}{ Image artifacts } & \multicolumn{2}{|c|}{$\begin{array}{l}\text { Diagnostic } \\
\text { confidence }\end{array}$} & \multicolumn{2}{|c|}{$\begin{array}{c}\text { Vascular } \\
\text { detectability }\end{array}$} & \multicolumn{2}{|c|}{$\begin{array}{l}\text { Image } \\
\text { impression }\end{array}$} \\
\hline & & AVG & STDV & AVG & STDV & AVG & STDV & AVG & STDV & AVG & STDV \\
\hline \multirow[t]{3}{*}{ Axial } & FBP & 3.61 & 0.49 & 3.05 & 0.81 & 3.18 & 0.76 & 2.82 & 0.99 & 1.66 & 1.12 \\
\hline & IMBR & 1.36 & 0.61 & 1.64 & 0.81 & 2.14 & 0.88 & 1.73 & 0.87 & 3.50 & 0.73 \\
\hline & IMBR + FBP & 1.39 & 0.58 & 1.68 & 0.83 & 1.68 & 0.67 & 1.50 & 0.73 & 2.82 & 0.84 \\
\hline \multirow[t]{3}{*}{ Coronal } & FBP & 3.27 & 0.66 & 2.64 & 0.87 & 2.77 & 0.77 & 2.39 & 0.84 & 1.43 & 0.79 \\
\hline & IMBR & 1.18 & 0.45 & 1.34 & 0.53 & 1.45 & 0.59 & 1.36 & 0.61 & 2.52 & 0.82 \\
\hline & IMBR + FBP & 1.32 & 0.56 & 1.41 & 0.66 & 1.43 & 0.66 & 1.32 & 0.67 & 1.98 & 0.82 \\
\hline
\end{tabular}




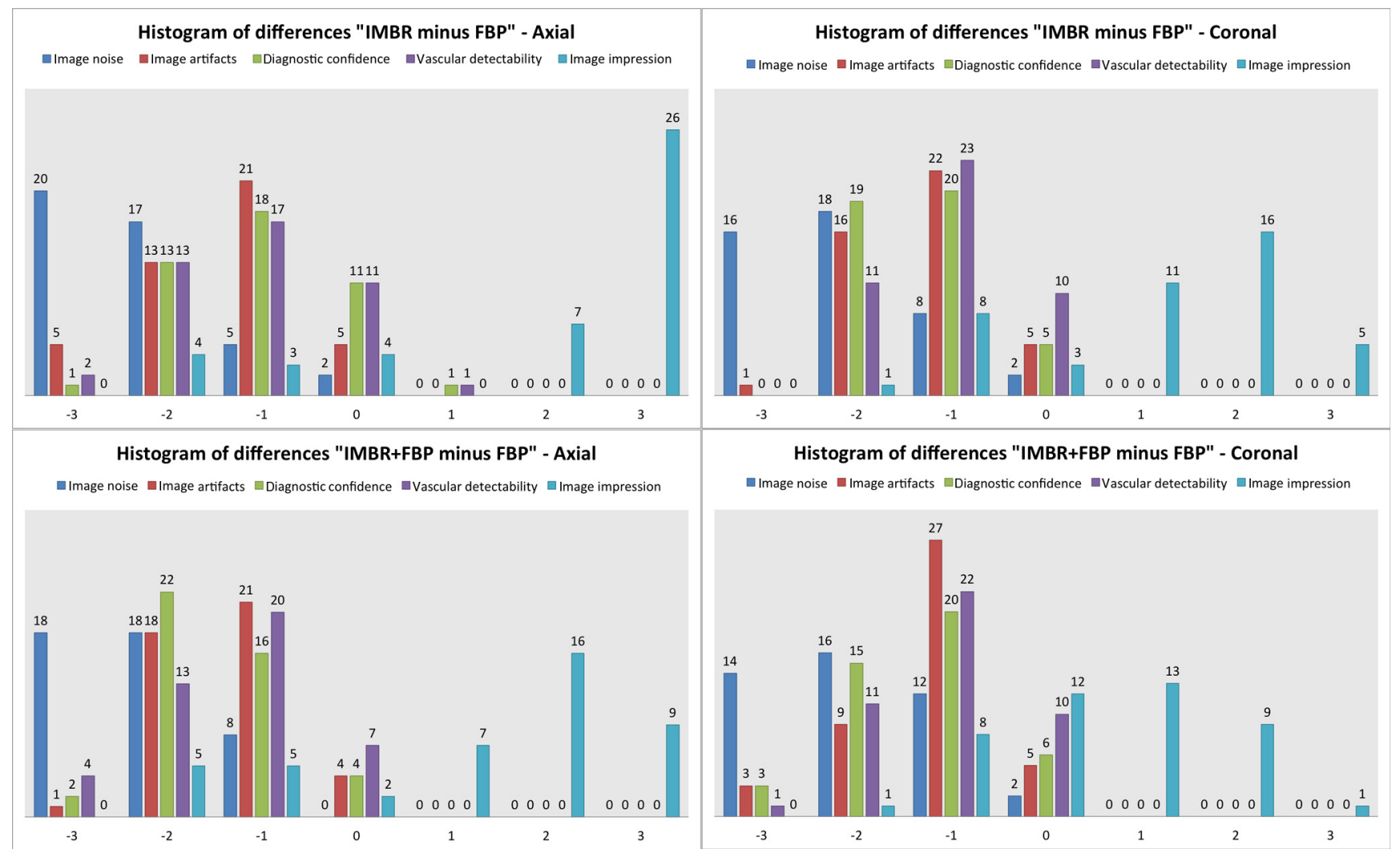

Fig. 4 The barplots of differences for "IMBR minus FBP"-axial (panel A),"IMBR minus FBP"-coronal (panel B), "IMBR + FBP minus FBP"- -axial (panel C), and "IMBR + FBP minus FBP"-coronal (panel $D$ ). Note that the absolute frequencies of observed differences are represented with respect to the reference values. (See text for details).

FBP"- coronal (Panel D). Note that for each subjective assessment metric, the total number of samples in each histogram is 44 (11 cases and 4 radiologists). One trend clearly visible in all panels is the superiority of IMBR-based reconstructions, with a single exception, namely image impression. Comparing IMBR and IMBR + FBP, we see that the image impression improves when using image fading. Further, we also see a better image impression in coronal than in axial-image orientation.

When considering all metrics, we see a similar trend; more small or zero differences were observed when considering IMBR versus IMBR + FBP or axial versus coronal. In other words, differences between FBP and IMBR are more pronounced in the axial than in the coronal orientation and are reduced in the combination IMBR + FBP. Clearly, if using FBP fading or a coronal orientation, the overall look and feel of the IMBR-based reconstruction is subjectively closer to the conventional FBP slices. While the image impression is not yet fully subjectively favored, diagnostic confidence and vascular detectability are superior in IMBR-based reconstruction. Note that these metrics are of higher importance when it comes to reliable diagnostics in the day-to-day routine. With regard to the classical image metrics of image noise and image artifacts, the IMBR-based reconstruction shows a significant increase in subjective image quality (see Table 1). Interobserver agreement for image noise, image artifacts, diagnostic confidence, vascular detectability, and image impression was very good with kappa values of $0.90,0.95,0.85,0.90$, and 0.95 , respectively.

These results could be confirmed in our CNR assessment, because for the aorta, paraspinal muscle, and subcutaneous fat comparable results were obtained. Mean image noise was lower with IMBR $(28 \mathrm{HU} \pm 26,17 \mathrm{HU} \pm 7$ and $11 \mathrm{HU} \pm 2$, respectively) and IMBR + FBP $(40 \mathrm{HU} \pm 23,26 \mathrm{HU} \pm 12$ and $19 \mathrm{HU} \pm 5$, respectively) than with $\mathrm{FBP}$ (267 $\mathrm{HU} \pm 54$, $172 \mathrm{HU} \pm 49$ and $104 \mathrm{HU} \pm 40$, respectively). CNR was higher with IMBR-based reconstruction compared to FBP for aorta versus paraspinal muscle and aorta versus subcutaneous fat (see Fig. 5). In numbers, CNR was $30 \pm 15$ and $61 \pm 20$ with IMBR, respectively, $19 \pm 10$ and $36 \pm 14$ with IMBR+ FBP, respectively, and $2.8 \pm 1.0$ and $6.8 \pm 2.3$ with FBP, respectively.

\section{Discussion}

In this study, we observed that when employing low-tubevoltage and low-tube-current, the IMBR algorithm enables a reduction of radiation exposure while providing CTA reconstructions with high diagnostic quality. IMBR is a model based fully iterative algorithm with additional edge-preserving regularization to trade-off noise and resolution. As previously presented by other investigations ${ }^{21-24}$ in low-tube-voltage CTA, a significant contrast enhancement of the vascular structure can be achieved when using iodine at $80 \mathrm{kVp}$ as a contrast media. A drawback of the low-tube-voltage technique is the increased image noise when keeping the tube current at similar levels as in high-tube-voltage protocols. The combination of $80 \mathrm{kVp}$ and IMBR illustrated an improved iodine signal and decreased image noise, which overcomes previous drawbacks with low-tube-voltage CTA. Subjective image assessment reveals that IMBR-based reconstruction techniques are superior to conventional FBP data, 


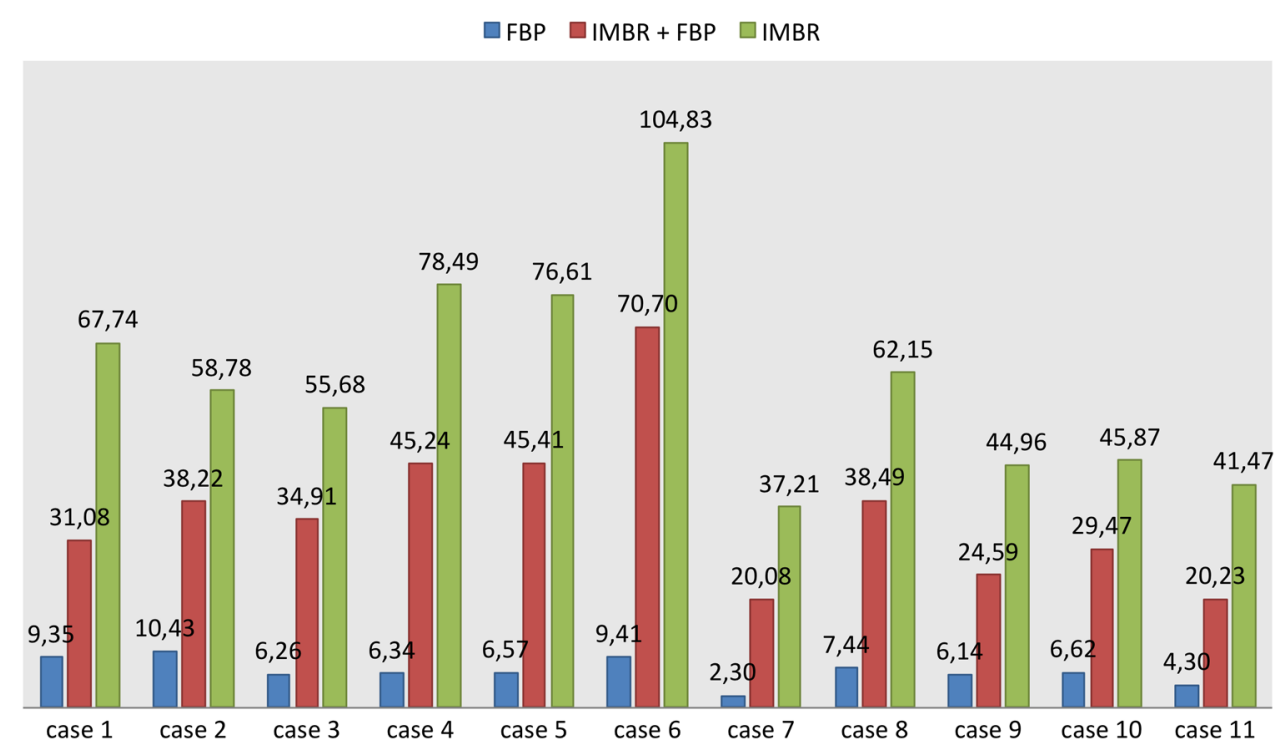

Fig. 5 Contrast-to-noise ratio (CNR) (aorta versus subcutaneous fat) for all patients and protocols. CNR is significantly higher with iterative model-based reconstruction (IMBR) compared to filtered backprojection (FBP) reconstructions.

especially with regard to diagnostic confidence. We illustrate that in low-dose CTAs, IMBR approaches are predominantly preferred. At the same time, the image impression of the IMBR reconstruction is currently not subjectively favored. We found in our systematic study that coronal images are favored over axial presentations. The reasons why coronal presentations are favored may be similar to already known CT phenomena. CT data are acquired using a helical or axial trajectory. These trajectories feature a dedicated rotation axis and it is well known and acknowledged that fundamental image parameters such as the spatial resolution and the noise power spectrum are quite different along the rotation axis than in axial images. Similar differences may be present in iteratively reconstructed images. We are not aware that this aspect has been studied systematically on clinical images so far. Here, we reported the results for the different metrics obtained on axial and coronal images separately.

Effective doses depended on BMI with an average of $3.0 \mathrm{mSv}$ compared to an average effective dose of about $12.0 \mathrm{mSv}$ for standard $120-\mathrm{kVp}$ acquisitions in our department. On this note, the diagnostic reference values, which are published by the German Federal Bureau of radiation safety, are $19.5 \mathrm{mSv}$ for this acquisition. In conclusion, we can report that we have improved image quality while significantly reducing radiation exposure. The dose aspect remains the highest of priorities in CT research. Our results illustrate that IMBR provides a promising dose-saving opportunity. Clearly, the potential exists for any clinical protocol to reduce radiation dose. However, the amount of reduction cannot be generalized to a fixed amount since the initial dose depends on the indication and protocol design, which may vary between different departments. Not only model-based algorithms but also other type of iterative algorithms for reconstruction of tomographic slices are popular in current CT research. Previous investigators ${ }^{5,10-15}$ have shown that there is a broad spectrum of commercial iterativereconstruction algorithms. At this point, we are not able to determine the absolute differences between these approaches because of missing detailed information.
There are drawbacks in our study design. Due to the limitations that appear in an initial investigation, we, for example, did not adjust the amount of contrast media. In future investigations, it will be necessary to determine the optimal amount of iodine when using IMBR and a low-tube-voltage protocol. Another drawback is the relatively small number of patients. The relative small number of patients already gives an important indicator toward dose reduction. However, the amount of reduction cannot be generalized for such a small number of patients, therefore, it will be necessary to conduct a larger or multicenter study for determining the optimal dose setting. ${ }^{25}$

One of the most important issues with regard to clinical translation of iterative reconstruction algorithms is the performance evaluation. Diagnostic quality is a combination of image quality and the indication of a given examination. For classical analytical image reconstruction algorithms, such as FBP, image quality can be measured with several different metrics, which include $\mathrm{CNR}$, image noise, resolution, and many more. It is important to comprehend that those metrics only provide limited informative value for images which are reconstructed with iterative algorithms (keyword: nonlinearity). Further, it is important that one recognizes that these metrics alone do not prove the diagnostic merit of a reconstruction. For example, one could use a strong image filter to completely eliminate image noise although such a filter would increasing blurring and would subsequently strongly limit the diagnostic quality. To determine if the reconstruction has diagnostic merit for a specific indication, one uses subjective image assessment. Thus, the indication of the examination in combination with all these metrics (objective and subjective) plays a major role in evaluating the diagnostic quality. Additionally, we have introduced the histogram of differences in order to remove interpatient variability and to some extent to remove the inter-reader variability.

We successfully demonstrated that IMBR significantly improves diagnostic quality and decreases radiation exposure at low-tube-voltages. As presented in our subjective image assessment, the image texture of IMBR reconstruction gives a different impression to the reader, which did not reduce 
diagnostic confidence. We strongly believe that if model-based algorithms such as IMBR can prove diagnostic merit, the subjective image impression will increase to current full dose FBP levels. In the meantime, a fallback option is fading the FBP images into the IMBR results. We think IMBR techniques will be a part of day-to-day clinical routine as simpler iterative approaches are today.

\section{Acknowledgments}

T.K. is employee of Philips GmbH; K.B. and S.Z. are employees of Philips Healthcare. The remaining authors have no financial disclosures and had complete, unrestricted access to the study data at all stages of the study.

\section{References}

1. W. H. Sommer et al., "Time-resolved CT angiography for the detection and classification of endoleaks," Radiology 263(3), 917-926 (2012).

2. C. Loewe et al., "64-Slice CT angiography of the abdominal aorta and abdominal arteries: comparison of the diagnostic efficacy of iobitridol $350 \mathrm{mgI} / \mathrm{ml}$ versus iomeprol $400 \mathrm{mgI} / \mathrm{ml}$ in a prospective, randomised, double-blind multi-centre trial," Eur. Radiol. 20(3), 572-583 (2010).

3. P. T. Johnson et al., "CT angiography with volume rendering: advantages and applications in splanchnic vascular imaging," Radiology 200(2), 564-568 (1996).

4. E. K. Fishman, "From the RSNA refresher courses: CT angiography: clinical applications in the abdomen," Radiographics 21, S3-S16 (2001).

5. D. Marin et al., "Low-tube-voltage, high-tube-current multidetector abdominal CT: improved image quality and decreased radiation dose with adaptive statistical iterative reconstruction algorithm: initial clinical experience," Radiology 254(1), 145-153 (2010).

6. A. Waaijer et al., "Circle of Willis at CT angiography: dose reduction and image quality-reducing tube voltage and increasing tube current settings," Radiology 242(3), 832-839 (2007).

7. S. T. Schindera et al., "Hypervascular liver tumors: low tube voltage, high tube current multi-detector row CT for enhanced detection-phantom study," Radiology 246(1), 125-132 (2008).

8. Y. Nakayama et al., "Abdominal CT with low tube voltage: preliminary observations about radiation dose, contrast enhancement, image quality, and noise," Radiology 237(3), 945-951 (2005).

9. D. Marin et al., "Hypervascular liver tumors: low tube voltage, high tube current multidetector CT during late hepatic arterial phase for detection: initial clinical experience," Radiology 251(3), 771-779 (2009).

10. P. B. Noël et al., "Initial performance characterization of a clinical noisesuppressing reconstruction algorithm for MDCT,' Am. J. Roentgenol. 197(6), 1404-1409 (2011).
11. A. Winklehner et al., "Raw data-based iterative reconstruction in body CTA: evaluation of radiation dose saving potential," Eur. Radiol. 21(12), 2521-2526 (2011).

12. H. C. Becker et al., "Radiation exposure and image quality of normal computed tomography brain images acquired with automated and organ-based tube current modulation multiband filtering and iterative reconstruction," Invest. Radiol. 47(3), 202-207 (2012).

13. B. M. Gramer et al., "Impact of iterative reconstruction on CNR and SNR in dynamic myocardial perfusion imaging in an animal model," Eur. Radiol. 22(12), 2654-2661 (2012).

14. B. K. Han et al., "Assessment of an iterative reconstruction algorithm (SAFIRE) on image quality in pediatric cardiac CT datasets," $J$. Cardiovasc. Comput. Tomogr. 6(3), 200-204 (2012).

15. T. Nakaura et al., "Low contrast agent and radiation dose protocol for hepatic dynamic CT of thin adults at 256-detector row CT: effect of low tube voltage and hybrid iterative reconstruction algorithm on image quality," Radiology 264(2), 445-454 (2012).

16. J. B. Thibault et al., "A three-dimensional statistical approach to improved image quality for multislice helical CT," Med. Phys. 34(11), 4526-4544 (2007).

17. J. A. Fessler, Statistical Image Reconstruction Methods for Transmission Tomography, SPIE Press, Bellingham, WA (2000).

18. K. M. Brown, S. Zabic, and T. Koehler, "Acceleration of ML iterative algorithms for CT by the use of fast start images," Proc. SPIE 8313, 831339 (2012).

19. F. Bergner et al., "Robust automated regularization factor selection for statistical reconstructions, in Proc. 2nd Int. Conf. on Image Formation in X-raz, Computed Tomography, pp. 267-270, Frederic Noo, Salt Lake City (2012).

20. C. McCollough, "The measurement, reporting, and management of radiation dose in CT," Report 96, American Association of Physicists in Medicine, Report of AAPM Task Group 23, pp. 1-28 (2008).

21. B. Wintersperger et al., "Aorto-iliac multidetector-row CT angiography with low kV settings: improved vessel enhancement and simultaneous reduction of radiation dose," Eur. Radiol. 15(2), 334-341 (2005).

22. A. B. Sigal-Cinqualbre et al., "Low-kilovoltage multi-detector row chest CT in adults: feasibility and effect on image quality and iodine dose," Radiology 231(1), 169-174 (2004).

23. H. Scheffel et al., "Low-dose CT coronary angiography in the stepand-shoot mode: diagnostic performance," Heart 94(9), 1132-1137 (2008).

24. Y. Nakayama et al., "Abdominal CT with low tube voltage: preliminary observations about radiation dose, contrast enhancement, image quality, and noise," Radiology 237(3), 945-951 (2005).

25. P. B. Noël et al., "Does iterative reconstruction lower CT radiation dose: evaluation of 15,000 examinations," PloS One 8(11), e81141 (2013).

Biographies of the authors are not available. 